PROCEEDINGS OF THE

AMERICAN MATHEMATICAL SOCIETY

Volume 125, Number 8, August 1997, Pages 2373-2378

S 0002-9939(97)03844-6

\title{
WEAK AMENABILITY AND THE SECOND DUAL OF THE FOURIER ALGEBRA
}

\author{
BRIAN FORREST
}

(Communicated by Theodore W. Gamelin)

\begin{abstract}
Let $G$ be a locally compact group. We will consider amenability and weak amenability for Banach algebras which are quotients of the second dual of the Fourier algebra. In particular, we will show that if $A(G)^{* *}$ is weakly amenable, then $G$ has no infinite abelian subgroup.
\end{abstract}

\section{INTRODUCTION}

In a recent paper Ghahramani, Loy and Willis [5] considered the possibility of the second dual of a Banach algebra being either amenable or weakly amenable. In particular, they were able to show that if $G$ is a locally compact group, then the second dual $L_{1}(G)^{* *}$ of the group algebra is amenable if and only if $G$ is finite [5, Theorem 1.3]. Moreover, they also succeeded in proving that if $G$ is abelian and $L_{1}(G)^{* *}$ is weakly amenable, then $G$ is discrete [5, Corollary 2.2]. In this note, we shall continue in the same spirit as in [5] by focusing on weak amenability for certain quotients of the second dual of the Fourier algebra $A(G)$ of $G$. By identifying $A(G)$ with $L_{1}(\widehat{G})$, we shall improve on the above results by showing that the second dual of an abelian group algebra is weakly amenable if and only if the group is finite.

\section{Preliminaries}

Let $G$ be a locally compact group. We let $A(G)$ denote the subspace of $C_{0}(G)$ consisting of functions of the form $u(x)=\sum_{i=1}^{\infty}\left(f_{i} * \tilde{g}_{i}\right)^{\wedge}(x)$ where $f_{i}, g_{i} \in L_{2}(G)$, $\sum_{i=1}^{\infty}\left\|f_{i}\right\|_{2}\left\|g_{i}\right\|_{2}<\infty, \widehat{f}(x)=f\left(x^{-1}\right)$ and $\tilde{f}(x)=\overline{f\left(x^{-1}\right)} . A(G)$ is a commutative Banach algebra with respect to pointwise operations and the norm

$$
\|u\|_{A(G)}=\inf \left\{\sum_{i=1}^{\infty}\left\|f_{i}\right\|_{2}\left\|g_{i}\right\|_{2} \mid u=\sum_{i=1}^{\infty}\left(f_{i} * \tilde{g}_{i}\right)^{\wedge}\right\}
$$

called the Fourier algebra of $G$ [3]. If $G$ is abelian, then $A(G)$ is simply the Fourier transform of the group algebra of $\widehat{G}$. $A(G)$ was introduced for noncommutative groups by Eymard in [3].

Let $V N(G)$ denote the closure of $L_{1}(G)$, considered as an algebra of convolution operators on $L_{2}(G)$, with respect to the weak operator topology on $B\left(L_{2}(G)\right)$. The von Neumann algebra $V N(G)$ can be identified with the dual of $A(G)$ [3].

Received by the editors June 5, 1995 and, in revised form, February 26, 1996.

1991 Mathematics Subject Classification. Primary 46H20; Secondary 43A20.

Key words and phrases. Fourier algebra, second dual, weakly amenable Banach algebra.

(C)1997 American Mathematical Society 
A subset $\mathcal{X}$ of $V N(G)$ is said to be topologically invariant if $u \cdot X \in \mathcal{X}$ for each $u \in A(G)$ and $X \in \mathcal{X}$ where $\langle u \cdot X, v\rangle=\langle X, v\rangle$. If $\mathcal{X}$ is a topologically invariant subspace of $V N(G)$, then $\mathcal{X}$ is said to be topologically introverted if for each $\Gamma \in \mathcal{X}^{*}$ and each $X \in \mathcal{X}$, we have $\Gamma \odot X \in \mathcal{X}$ where $\Gamma \odot X$ is the functional on $A(G)$ defined by $\langle\Gamma \odot X, u\rangle=\langle\Gamma, u \cdot X\rangle$. If $\mathcal{X}$ is topologically introverted, then $\mathcal{X}^{*}$ is a Banach algebra with respect to the product $\Phi \odot \Gamma$ where $\langle\Phi \odot \Gamma, X\rangle=\langle\Phi, \Gamma \odot X\rangle$. In case $\mathcal{X}=V N(G)$, the product on $V N(G)^{*}=A(G)^{* *}$ is simply one of the Arens products introduced in [1]. Moreover, if $\mathcal{X}$ is topologically introverted and $\widetilde{\Gamma}$ and $\widetilde{\Phi}$ are extensions of $\Gamma$ and $\Phi$ respectively, then $\widetilde{\Phi} \odot \widetilde{\Gamma}$ extends $\Phi \odot \Gamma$. For this reason, we may view $\mathcal{X}^{*}$ as a quotient algebra of $A(G)^{* *}$. We refer the reader to $[14, \S 5]$ for further details.

Let $C_{\lambda}^{*}(G)$ and $C_{\delta}^{*}(G)$ denote respectively the $C^{*}$-subalgebras of $V N(G)$ generated by $L_{1}(G)$ viewed as left convolution operators and by $G$ viewed as left translation operators on $L_{2}(G)$ via the left regular representation $\lambda$. $C^{*}(G)$, the group $C^{*}$-algebra of $G$, is the enveloping $C^{*}$-algebra of $L_{1}(G)$. The dual of $C^{*}(G)$ is $B(G)$, the Fourier-Stieltjes algebra of $G$. The dual of $C_{\lambda}^{*}(G)$ is $B_{\lambda}(G)$, a closed ideal in $B(G)$. Moreover, $B(G)=B_{\lambda}(G)$ precisely when the group $G$ is amenable. The dual of $C_{\delta}^{*}(G)$ is denoted by $B_{\delta}\left(G_{d}\right)$ and is a closed subalgebra of $B\left(G_{d}\right)$, the Fourier-Stieltjes algebra of $G$ viewed as a discrete group. It should be noted that $B_{\delta}\left(G_{d}\right)$ contains $B_{\lambda}\left(G_{d}\right)$ and hence $A\left(G_{d}\right)$.

A Banach algebra $\mathcal{A}$ is said to be amenable if every continuous derivation $D$ : $\mathcal{A} \rightarrow X^{*}$ is inner whenever $X$ is a Banach $\mathcal{A}$-bimodule. $\mathcal{A}$ is weakly amenable if every continuous derivation $D: \mathcal{A} \rightarrow \mathcal{A}^{*}$ is inner.

\section{Weak amenability of $A(G)^{* *}$}

Let $\mathcal{X}$ be a topologically introverted subspace of $V N(G)$. If $\mathcal{X}$ contains $C_{\delta}^{*}(G)$, we let

$$
K_{\delta}(\mathcal{X})=\left\{\Gamma \in \mathcal{X}^{*} \mid\langle\Gamma, \lambda(g)\rangle=0 \text { for every } g \in G\right\}
$$

where $\lambda(g)$ is the left translation operator on $L_{2}(G)$ determined by $g$ via the left regular representation $\lambda$. As in the remarks preceding Theorem 3.6 of [13], we have that $K_{\delta}(\mathcal{X})$ is a weak- ${ }^{*}$ closed two-sided ideal of $\mathcal{X}^{*}$. Similarly, if $\mathcal{X}$ contains $C_{\lambda}^{*}(G)$, then $K_{\lambda}(\mathcal{X})=\left\{\Gamma \in \mathcal{X}^{*} \mid\langle\Gamma, \lambda(f)\rangle=0\right.$ for every $\left.f \in L_{1}(G)\right\}$ is also a weak-* closed ideal in $\mathcal{X}^{*}$.

Let $U C B(\widehat{G})$ denote the closed linear span of $A(G) \cdot V N(G)$. Then $U C B(\widehat{G})$ is a topologically introverted $C^{*}$-subalgebra of $V N(G)$ (see [14, Proposition 5.2]). Given $X \in V N(G)$, let $\mathcal{O}(X)=\left\{u \cdot X \mid u \in A(G),\|u\|_{A(G)} \leq 1\right\} . X$ is (weakly) almost periodic if $\mathcal{O}(X)$ is relatively (weakly) compact. Let $W A P(\widehat{G})$ and $A P(\widehat{G})$ denote the spaces of weakly almost periodic and almost periodic functionals on $A(G)$ respectively. $A P(\widehat{G})$ and $W A P(\widehat{G})$ are also topologically introverted subspaces of $V N(G)$. Moreover, each of the spaces $U C B(\widehat{G}), W A P(\widehat{G})$ and $A P(\widehat{G})$ contain $C_{\delta}^{*}(G)$ as a closed subspace, while $U C B(\widehat{G})$ and $W A P(\widehat{G})$ contain $C_{\lambda}^{*}(G)$.

The following lemma can be obtained by modifying the arguments leading to [13, Theorem 3.5], and [13, Theorem 3.6].

Lemma 3.1. Let $\mathcal{X}$ be a topologically introverted subspace of $V N(G)$ which contains $C_{\delta}^{*}(G)$. Then $\mathcal{X}^{*} / K_{\delta}(\mathcal{X})$ is isometrically isomorphic to $B_{\delta}\left(G_{d}\right)$. Let $\mathcal{X}$ be a topologically introverted subspace of $V N(G)$ which contains $C_{\lambda}^{*}(G)$. Then $\mathcal{X}^{*} / K_{\lambda}(\mathcal{X})$ is isometrically isomorphic to $B_{\lambda}(G)$. 
Theorem 3.2. Let $\mathcal{X}$ be a topologically introverted subspace of $V N(G)$ which contains $C_{\delta}^{*}(G)$. If $\mathcal{X}^{*}$ is weakly amenable, then every abelian subgroup $H$ (not necessarily closed) of $G$ is finite. Moreover, $G$ is totally disconnected.

Proof. It follows from Lemma 3.1 that $B_{\delta}\left(G_{d}\right)$ is a quotient of $\mathcal{X}^{*}$.

Consider $H$ as a subgroup of $G_{d}$. Let $1_{H}$ denote the characteristic function of $H$. Then $1_{H} \in B\left(G_{d}\right)$ [3]. Moreover, $1_{H} B_{\delta}\left(G_{d}\right)$ is isometrically isomorphic to a weak-* closed subalgebra of $B\left(H_{d}\right)$ which contains $A\left(H_{d}\right)$. Since $H$ is abelian and therefore amenable as a discrete group, $1_{H} B_{\delta}\left(G_{\delta}\right)$ is isometrically isomorphic to $B\left(H_{d}\right)$. Thus $B\left(H_{d}\right)$ is also a homomorphic image of $\mathcal{X}^{*}$.

If $H$ is infinite, then $\left(H_{d}\right)^{\wedge}$ is nondiscrete. It follows from [2] that the measure algebra $M\left(\left(H_{d}\right)^{\wedge}\right)$ has a nonzero point derivation $D$. However, since $M\left(\left(H_{d}\right)^{\wedge}\right)$ is a homomorphic image of $\mathcal{X}^{*}, D$ would lift to $\mathcal{X}^{*}$. This is impossible if $\mathcal{X}^{*}$ is assumed to be weakly amenable.

Let $G_{0}$ denote the connected component of $e$. Then since $G$ is periodic, so is $G_{0}$. Moreover, the same is true for any homomorphic image of $G_{0}$. Let $U$ be a neighborhood of $e$ in $G_{0}$. Then there exists a compact normal subgroup $N \subseteq U$ such that $G_{0} / N$ is a periodic, connected Lie group. Therefore $G_{0} / N$ is trivial. Since this is true for all such $U, G_{0}$ must be trivial.

When $G$ is abelian, $C_{\delta}^{*}\left(G_{d}\right)$ is simply the Fourier transform of $A P(\widehat{G})$ which, abusing the above notation, is the space of almost periodic functions on the dual group $\widehat{G}$. The following improves [5, Corollary 2.2]:

Corollary 3.3. Let $G$ be an abelian locally compact group. Let $\mathcal{X}$ be a topologically introverted subspace of $L_{\infty}(G)$ which contains $A P(G)$. If $\mathcal{X}^{*}$ is weakly amenable, then $G$ is finite. In particular, if $L_{1}(G)^{* *}$ is weakly amenable, then $G$ is finite.

We have seen that, as an immediate consequence of Theorem 3.2, we get that if $A(G)^{* *}$ is weakly amenable, then $G$ is periodic. That is, every element of $G$ has finite order. Perhaps the most significant class of periodic groups is the locally finite groups. It is well known that an infinite locally finite group always contains an infinite abelian subgroup. The same is true for infinite locally solvable groups and infinite periodic elementary groups (see [12] for properties of these groups). Indeed we have:

Corollary 3.4. Let $G$ be a locally compact group which, when viewed as a discrete group, satisfies one of the following conditions:

(i) $G$ is locally finite;

(ii) $G$ is an elementary group;

(iii) $G$ is locally solvable;

(iv) $G$ is isomorphic to a subgroup of $\mathrm{GL}(n, \mathbb{F})$ for some $n$ and any field $\mathbb{F}$.

If $\mathcal{X}$ is a topologically introverted subspace of $V N(G)$ which contains $C_{\delta}^{*}\left(G_{d}\right)$, then $\mathcal{X}^{*}$ is weakly amenable if and only if $G$ is finite.

Proof. Clearly, if $G$ is finite then $\mathcal{X}^{*}=V N(G)^{*}$ is weakly amenable.

Conversely, if $G$ is infinite and either locally finite or locally solvable, then $G$ has an infinite abelian subgroup. If $G$ is periodic and elementary or periodic and linear, then $G$ is locally finite (see [4, Proposition 3]). It now follows from Theorem 3.2 that $\mathcal{X}^{*}$ cannot be weakly amenable.

Recall that a discrete group $G$ has polynomial growth if for every finite set $F \subseteq G$ there exists a $p \in \mathbb{N}$ such that $\left|F^{n}\right|=O\left(n^{p}\right)$. 
Corollary 3.5. Let $G$ be a locally compact group. Let $H$ be a subgroup of $G$ which, when viewed as a discrete group, has polynomial growth. If $\mathcal{X}$ is a topologically introverted subspace of $V N(G)$ which contains $C_{\delta}^{*}\left(G_{d}\right)$, and $\mathcal{X}^{*}$ is weakly amenable, then $H$ is finite.

Proof. Let $\Gamma$ be a finitely generated subgroup of $H_{d}$. As $H_{d}$ is discrete, $\Gamma$ also has polynomial growth. Therefore, $\Gamma$ must have a nilpotent subgroup $\Gamma_{1}$ which is of finite index [9]. If $\Gamma_{1}$ is infinite, it must contain an infinite abelian subgroup. Since this is impossible by Theorem $3.2, \Gamma$ is finite. This means that $H_{d}$ is locally finite. Since infinite locally finite groups have infinite abelian subgroups, $H_{d}$ is finite.

Proposition 3.6. Let $\mathcal{X}$ be a topologically introverted subspace of $W A P(\widehat{G})$ which contains $C_{\lambda}^{*}(G)$. If $\mathcal{X}^{*}$ is weakly amenable, then both $B_{\lambda}(G)$ and $A(G)$ are weakly ameanble.

Proof. It follows from Lemma 3.1 that $B_{\lambda}(G)$ is a quotient of $\mathcal{X}^{*}$. Since $\mathcal{X}$ is contained in $W A P(\widehat{G}), \mathcal{X}^{*}$ is a commutative Banach algebra [14, Theorem 5.6]. Thus $B_{\lambda}(G)$ is weakly amenable. $A(G)$ is a closed ideal in $B_{\lambda}(G)$, and since the empty set is a set of spectral synthesis for $A(G), A(G)^{2}$ is dense in $A(G)$. It follows that $A(G)$ is also weakly amenable by [10, Proposition 2.2$]$.

\section{Amenability of $A(G)^{* *}$}

In light of the results of the previous section, it seems reasonable to conjecture that if $A(G)^{* *}$ is weakly amenable, then $G$ is finite. However, there are groups such as the Tarski Monster, an infinite group for which each proper subgroup is finite and for which it may still be possible that $A(G)^{* *}$ is weakly amenable. In this section, we will show that under the stronger hypothesis that $A(G)^{* *}$ be amenable, then $G$ must be finite for a class of groups which contains all discrete groups and all locally compact groups such that $\overline{[G, G]}$ is not open.

We refer the reader to [16] for analogous results for group and semigroup algebras.

Theorem 4.1. Let $\mathcal{X}$ be a topologically introverted subspace of $V N(G)$ which contains $C_{\delta}^{*}(G)$. If $\mathcal{X}^{*}$ is amenable, then both $A\left(G_{d}\right)$ and $B\left(G_{d}\right)$ are amenable. In particular, $G$ is amenable as a discrete group.

Proof. Lemma 3.1 implies that $B_{\delta}\left(G_{d}\right)$ is a quotient of $\mathcal{X}^{*}$ and is therefore also amenable. Since $A\left(G_{d}\right)$ is a complemented ideal of $B_{\delta}\left(G_{d}\right), A\left(G_{d}\right)$ is amenable as well. This clearly implies that $G$ is amenable as a discrete group. Finally, since $G_{d}$ is amenable, $B_{\delta}\left(G_{d}\right)=B\left(G_{d}\right)$.

Lemma 4.2. Let $\mathcal{X}$ be a topologically introverted subspace of $V N(G)$ which contains $C_{\lambda}^{*}(G)$. If $\mathcal{X} \subseteq U C B(\widehat{G})$, then $\mathcal{X}^{*}=B_{\lambda}(G) \oplus K_{\lambda}(\mathcal{X})$.

Proof. In [15, §4], Lau and Losert constructed an embedding $\pi$ of $B_{\lambda}(G)$ into $U C B(\widehat{G})^{*}$ with $\pi(u)$ being the unique extension of $u \in B_{\lambda}(G)=\left(C_{\lambda}^{*}(G)\right)^{*}$ with the same norm. We can define a continuous projection $p$ from $U C B(\widehat{G})^{*}$ onto $K_{\lambda}(U C B(\widehat{G}))$ as follows:

For $\Gamma \in U C B(\widehat{G})^{*}$, let $u_{\Gamma}$ be the restriction of $\Gamma$ to $C_{\lambda}^{*}(G)$. Then $P(\Gamma)=$ $\Gamma-\pi\left(u_{\Gamma}\right)$ is the desired projection.

For $\mathcal{X} \subseteq U C B(\widehat{G})$, the argument is identical. 
Lemma 4.3. Let $\mathcal{X}$ be a topologically introverted subspace of $V N(G)$ which contains $C V_{\lambda}^{*}(G)$. If $\mathcal{X} \subseteq U C B(\widehat{G})$ and $\mathcal{X}^{*}$ is amenable, $K_{\lambda}(\mathcal{X})$ has an identity $E$ which is contained in the center of $\mathcal{X}^{*}$.

Proof. We follow the proof of [5, Theorem 1.3].

By Lemma $4.2, K_{\lambda}(\mathcal{X})$ is a complemented ideal in $\mathcal{X}^{*}$ and is therefore amenable. As such, $K_{\lambda}(\mathcal{X})$ has a bounded approximate identity $\left\{e_{\alpha}\right\}$ which we may assume converges weak- ${ }^{*}$ to $E \in K_{\lambda}(\mathcal{X})$. Weak-* continuity in the first variable shows that $E$ is a left identity. If $\Gamma \in K_{\lambda}(\mathcal{X})$, then $\Gamma E=\lim _{\alpha}(\Gamma E) e_{\alpha}=\lim \Gamma\left(E e_{\alpha}\right)=\Gamma$, so that $E$ is in fact a two-sided identity for $K_{\lambda}(\mathcal{X})$. That $E$ is central follows since $K_{\lambda}(\mathcal{X})$ is a two-sided ideal of $\mathcal{X}^{*}$.

Theorem 4.4. Let $G$ be such that $A(G)^{* *}$ is amenable. Then $G$ is compact.

Proof. If $A(G)^{* *}$ is amenable, then it has a bounded approximate identity. It follows from [5, Lemma 1.1] that $A(G)^{* *}$ has an identity. That $G$ is compact is [13, Proposition 3.2 (b)].

Theorem 4.5. Let $G$ be a second countable locally compact group for which $\overline{[G, G]}$ is not open. If $\operatorname{UCB}(\widehat{G})^{*}$ is amenable, then $G$ is discrete.

Proof. Lemma 4.3 shows that $K_{\lambda}(U C B(\widehat{G}))$ has an identity $E$ which is central in $U C B(\widehat{G})^{*}$. It follows from [15, Lemma 5.7] that $E=0$. Thus $K_{\lambda}(U C B(\widehat{G}))=\{0\}$ and $C_{\lambda}^{*}(G)=U C B(\widehat{G})$. Finally, that $G$ is discrete follows from [8, Proposition 4.5].

Corollary 4.6. Let $G$ be a locally compact group which satisfies one of the following conditions

(i) $G$ is discrete.

(ii) $G$ is abelian.

(iii) $G$ is a second countable group for which $\overline{[G, G]}$ is not of finite index in $G$. If $A(G)^{* *}$ is amenable, then $G$ is finite.

Proof. Case (i) follows immediately from Theorem 4.4. Case (ii) is [5, Theorem 1.3]. It also follows from Corollary 3.3.

Case (iii): If $A(G)$ is ameanble, Theorem 4.4 shows that $G$ is compact and hence that $A(G)^{* *}=U C B(\widehat{G})$. That $G$ is finite now follows immediately from Theorem 4.5.

We wish to note that at present we now of no locally compact group $G$ for which $A(G)$ is amenable other than those which are finite extensions of abelian groups. Indeed, recent work of B. Johnson [11] has provided us with a significant number of compact groups for which $A(G)$ is not even weakly amenable.

We will conclude with a few remarks concerning related questions for ideals in $A(G)$.

Suppose that $I$ is a closed ideal of $A(\mathbb{Z})$ and that $I^{* *}$ is amenable. By a result of Gourdeau [6] (see [5, Theorem 1.8] for a short proof), it follows that $I$ must also be amenable. Since $I$ would then possess a bounded approximate identity, it is well known that $I=I(A)$ where $A$ is an element of the coset ring of $\mathbb{Z}$ [17]. If $I$ is infinite dimensional, then a simple induction argument can be used to show that there exists an infinite subgroup $H$ of $\mathbb{Z}$ such that, with the exception of at most finitely many points, $x H$ is contained in $G \backslash A$ for some coset $x H$ of $H$. Furthermore, a little thought shows that the restriction of $A(\mathbb{Z})$ to $x H$ yields an algebra which 
is isometrically isomorphic to $A(H)$ and is such that its second dual is amenable. Since this is clearly impossible, $I^{* *}$ can only be amenable if $I$ is finite dimensional. We would speculate that an analogous statement would hold true for any discrete group. In contrast, we have the following proposition which we compare with [5, Proposition 1.01], where it is shown that an amenable algebra which is a Hilbert space must be finite dimensional.

Proposition 4.7. let $G$ be a discrete amenable group. Then $A(G)$ has an infinite dimensional closed ideal I which is isometrically isomorphic with a Hilbert space and is such that $I=I^{* *}$ is weakly amenable.

Proof. That $A(G)$ has a closed ideal $I$ which is isomorphic to a Hilbert space is [7, Theorem 5(b)]. Since $G$ is amenable, $A(G)$ is a Ditkin algebra. It follows that every subset of $G$ is a set of spectral synthesis. As such, $I$ is idempotent. Since $G$ is discrete, $A(G)$ is weakly amenable. Therefore, $I=I^{* *}$ is also weakly amenable.

\section{REFERENCES}

1. R. Arens, The adjoint of a bilinear operation, Proc. Amer. Math. Soc., 2 (1951), 839-848. MR 13:659f

2. G. Brown and W. Moran, Point derivations on $M(G)$, Bull. London Math. Soc., 8 (1976), 57-64. MR 54:5744

3. P. Eymard, L'algèbre de Fourier d'un groupe localement compact, Bull. Soc. Math. France, 92 (1964), 181-236. MR 37:4208

4. B. Forrest, Arens regularity and the $A_{p}(G)$ algebras, Proc. Amer. Math. Soc., 119 (1991), 595-598. MR 93k:43003

5. F. Ghahramani, R. J. Loy and G. A. Willis, Amenability and weak amenability of second conjugate Banach algebras, Proc. Amer. Math. Soc. 124 (1996), 1489-1497. MR 96g:46036

6. F. Gourdeau, Amenability of Banach algebras, Ph.D. thesis, University of Cambridge, 1989.

7. E. E. Granirer, On some properties of the Banach algebras $A_{p}(G)$ for locally compact groups, Proc. Amer. Math. Soc., 95 (1985), 375-381. MR 87e:43005

8. _ Density theorems for some linear subspaces and some $C^{*}$-subalgebras of $V N(G)$, Sympos. Math. INDAM, vol. XXII, Academic Press, New York, 1977, pp. 61-70. MR 58:6935

9. M. L. Gromov, Groups of polynomial growth and expanding maps, Inst. Hautes Études Sci. Publ. Math., 53 (1981), 53-73. MR 83b:53041

10. N. Grønbaek, A characterization of weakly amenable Banach algebras, Studia Math., 94 (1989), 149-162. MR 92a:46055

11. B. Johnson, Nonamenability of the Fourier algebra for compact groups, J. London Math. Soc., 50 (1994), 361-374. MR 95i:43001

12. O. Kegel and B. Wehrfritz, Locally Finite Groups, Wiley, New York, 1984. (2nd ed. of MR $57: 9848)$

13. A. T. Lau, The second conjugate algebra of the Fourier algebra of a locally compact group, Trans. Amer. Math. Soc., 267 (1981), 53-63. MR 83e:43009

14. _ Uniformly continuous functionals on the Fourier algebra of any locally compact group, Trans. Amer. Math. Soc., 251 (1979), 39-59. MR 80m:43009

15. A. T. Lau and V. Losert, The $C^{*}$-algebra generated by operators with compact support on a locally compact group, J. Funct. Anal., 112 (1993), 1-30. MR 94d:22005

16. A. T. Lau and R. J. Loy, Amenable convolution algebras, preprint.

17. H. Rosenthal, Projections onto translation invariant subspaces of $L^{p}(G)$, Mem. Amer. Math. Soc. No. 63 (1966). MR 35:2080

Department of Pure Mathematics, University of Waterloo, Waterloo, Ontario, CANADA N2L 3G1 